\title{
EFEITO DE DIFERENTES CLASSES DE ALTURA E INTENSIDADES DE DESRAMA ARTIFICIAL SOBRE O CRESCIMENTO DE UM HÍBRIDO DE EUCALIPTO
}

\author{
Diego Piva Cezana ${ }^{1}$, José Franklim Chichorro ${ }^{2}$, Leandro Tose Martins ${ }^{1}$, Thiago Reggiani Cotta $^{1}$, \\ Jeferson Leal Silva ${ }^{1}$ \\ ${ }^{1}$ Eng. Florestal, Mestrando em Ciências Florestais, UFES, Jerônimo Monteiro, ES, Brasil - diegocezana@yahoo.com.br; \\ ltosemartins@hotmail.com; thiagoreggiani@ hotmail.com; jefersonlealsilva@yahoo.com.br \\ ${ }^{2}$ Eng. Florestal, Dr., Depto. de Engenharia Florestal, UFES, Jerônimo Monteiro, ES, Brasil - jfufes@ gmail.com
}

Recebido para publicação: 21/09/2010 - Aceito para publicação: 01/12/2011

\begin{abstract}
Resumo
O presente trabalho teve como objetivo avaliar o efeito da interação entre a intensidade de desrama e a classe de altura sobre o crescimento do híbrido Eucalyptus grandis $x$ Eucalyptus urophylla. $\mathrm{O}$ experimento foi instalado em uma área declivosa no município de Mimoso do Sul, ES. Foi utilizado o delineamento inteiramente casualizado em arranjo fatorial 4 x 4 (classes de altura $\mathrm{x}$ intensidade de desrama). Ficou demonstrado que tanto a classe de altura quanto a intensidade de desrama tiveram efeito significativo sobre o crescimento das árvores ao nível de 5\% de probabilidade, indicando que árvores maiores que 4 metros de altura apresentam crescimento mais intenso que árvores situadas em estratos inferiores a este. Com o aumento da intensidade de desrama, notou-se uma redução do crescimento, sendo o tratamento com intensidade de $80 \%$ de desrama o que apresentou menores acréscimos de diâmetro e altura. Conclui-se que a interação da intensidade de desrama com a classe de altura das árvores não apresenta efeito significativo sobre o crescimento ao nível de 5\% de probabilidade.

Palavras-chave: Tratos silviculturais; poda; produção florestal.
\end{abstract}

\begin{abstract}
Effect of different classes of height and pruning intensity on growth of a hybrid eucalyptus. This study aimed to evaluate the effect of interaction between pruning intensity and height class on the growth of the hybrid Eucalyptus grandis x Eucalyptus urophylla. The experiment was installed on a sloping area in the municipality of Mimoso do Sul, ES. The design used was the completely randomized in factorial arrangement $4 \times 4$ (classes of height $x$ pruning intensity). That revealed that as the classes of height as the pruning intensity had significant effect on tree growth at $5 \%$ probability, indicating that trees larger than 4 meters of height had more intense growth than trees located in lower stratus and with increase of pruning intensity; there were a reduction on the growth, and the $80 \%$ pruning treatment presented smaller diameter and height growth. Interaction between pruning intensity and height class on the trees' growth was not significant at $5 \%$ probability.

Keywords: Silvicultural treatment; pruning; forest production.
\end{abstract}

\section{INTRODUÇÃO}

É notável nos últimos anos o crescente aumento da demanda por madeiras para serraria provenientes de áreas de reflorestamento, principalmente dos gêneros Pinus e Eucalyptus. A busca pelo melhor aproveitamento das florestas e de melhor remuneração pela madeira tem incentivado, por parte de empresas, estratégias para oferta de madeira serrada de Eucalyptus sp. (VALE et al., 2002). As empresas que visam tal produção investem no manejo de suas florestas com a finalidade de aumentar a qualidade da madeira. Assim, a desrama artificial é uma técnica que tem a finalidade de diminuir a incidência de nós e com isso agregar valor à floresta, produzindo um material de alta qualidade, livre de defeitos e, principalmente, com maior resistência, durabilidade e beleza (SCHNEIDER et al., 1999; VALE, 2000; 
FINGER et al., 2001; PIRES et al., 2002; PULROLNIK, 2002; ALMEIDA, 2003; HOPPE; FREDDO, 2003).

De acordo com Schettino et al. (2000), principalmente nas décadas de 60 e 70, o setor florestal madeireiro no estado do Espírito Santo tinha destaque. Entretanto, como a exploração dos recursos naturais era feita de forma desordenada, houve esgotamento das reservas. Isso fez com que o estado perdesse expressão no setor de serrarias, que migraram principalmente para o sul da Bahia. Ainda segundo o autor, caso surjam investimentos no setor, o estado pode reverter o quadro de escassez de matéria-prima para serraria em médio prazo.

No estado do Espírito Santo, o reflorestamento com eucalipto foi intensificado com a instalação de empresas consumidoras da matéria-prima madeireira, como a indústria de celulose, metalúrgicas e olarias, bem como pelo consumo doméstico. O uso de espécies do gênero Eucalyptus, em particular, está relacionado com sua fácil adaptação às condições edafoclimáticas da região, ao seu rápido crescimento, produtividade e alternativas de uso e de mercado. Esse cenário é confirmado pela área plantada com eucalipto no estado, que é de 204,57 mil ha (ASSOCIAÇÃO BRASILEIRA DOS PRODUTORES DE FLORESTA PLANTADA (ABRAF), 2010). Especificamente nesse estado, o setor florestal merece destaque, pois possui localização geográfica privilegiada e adequada infraestrutura de transportes marítimo, ferroviário e rodoviário (SCHETTINO et al., 2000).

Quanto a fatores que influenciam a produtividade, podem-se considerar a qualidade das mudas, $\mathrm{o}$ preparo adequado do solo, a adubação inicial e de cobertura e os tratos silviculturais, como a desrama artificial.

Por isso, o conhecimento do efeito dos fatores de produção da espécie é relevante quando relacionados à produtividade e à qualidade da madeira (HOSOKAWA et al., 1998), bem como quando relacionados ao custo/benefício. Assim, a definição de alternativas que aumentem a eficácia e a eficiência no uso dos fatores de produção possibilitará aos produtores implantar projetos de investimentos com maior estabilidade de produção e retorno econômico (REZENDE et al., 2006).

Entre os tratos silviculturais utilizados nos plantios, a desrama artificial merece destaque, pois com ela é possível melhorar as propriedades físicas da madeira, quando seu destino está programado para serraria, reduzindo a quantidade de madeira com presença de nós, obtendo produtos mais nobres e agregando valor ao produto final (HOSOKAWA et al., 1998). No entanto, a redução da área fotossinteticamente ativa da planta pode prejudicar seu crescimento em diâmetro, altura e consequentemente em volume. Desse modo, são necessários trabalhos que definam a melhor intensidade e época de utilização dessa técnica, para que se produza madeira de qualidade sem reduzir a taxa de crescimento da planta.

Nesse sentido, o presente estudo teve como hipóteses que o aumento da intensidade de desrama artificial proporciona uma redução do crescimento em diâmetro e altura das árvores, e que as árvores das maiores classes de altura têm crescimento mais intenso que as árvores localizadas em extratos inferiores.

\section{Objetivo geral}

O objetivo geral do presente trabalho foi verificar o efeito da interação entre a intensidade de desrama artificial e a classe de altura sobre o crescimento do híbrido Eucalyptus grandis x E. urophylla.

\section{Objetivos específicos}

Os objetivos específicos do trabalho foram:

a) Avaliar o efeito da intensidade de desrama no crescimento em altura e diâmetro das árvores através de medições sucessivas dessas variáveis aos 24 e 30 meses.

b) Avaliar o efeito da classe de altura no crescimento em altura e diâmetro das árvores através de medições sucessivas dessas variáveis aos 24 e 30 meses.

c) Avaliar o efeito da interação intensidade de desrama x classe de altura da árvore no crescimento em diâmetro e altura das árvores através de medições sucessivas dessas variáveis aos 24 e 30 meses.

d) Avaliar possíveis alterações da condição fitossanitária e seus efeitos em decorrência da intensidade de desrama e classes de altura através de observação das árvores selecionadas aos 24 e 30 meses de idade.

e) Identificar a intensidade adequada de desrama para o híbrido Eucalyptus grandis x E. urophylla. 


\section{REVISÃO BIBLIOGRÁFICA}

Desrama é o fenômeno de queda e/ou retirada de galhos e/ou folhas do tronco de uma árvore. Ela pode ocorrer de forma natural, devido à ação de agentes físicos ou bióticos do meio, ou de forma artificial, quando há interferência do homem. A desrama artificial é a retirada dos galhos de uma porção do tronco de uma árvore, de maneira mecânica. Geralmente se produz madeira de melhor qualidade e livre de nós quando se utiliza essa técnica (SCHNEIDER et al., 1999; FINGER et al., 2001). Vários trabalhos foram realizados sobre desrama artificial, os quais enfatizam, principalmente, o efeito da intensidade de desrama sobre o crescimento das árvores, a fim de se encontrar a melhor forma de aplicação da técnica. Geralmente os trabalhos sobre desrama consideram a quantidade de galhos e/ou folhas retirados do tronco de acordo com uma porcentagem em relação à altura total da árvore (SCHNEIDER et al., 1999; FINGER et al., 2001; PIRES et al., 2002; HOPPE; FREDDO, 2003), ou de acordo com a altura de desrama em relação ao nível do solo (PULROLNIK, 2002; ALMEIDA, 2003; POLLI, 2005; MONTE et al., 2009).

A retirada de ramos vivos da árvore reduz a superfície fotossinteticamente ativa, além de limitar o processo de respiração. Ao se retirar os ramos inferiores, que geralmente têm uma participação menor na taxa de fotossíntese realizada pela planta, pode-se estar contribuindo para reduzir o consumo de reservas pela respiração (KRAMER; KOZLOWSKI, 1972). Entretanto, caso a desrama seja realizada de forma muito severa, retirando-se muitas folhas, pode ser que venha a prejudicar o crescimento da árvore, justamente pela redução drástica da superfície fotossintética da planta (PULROLNIK, 2002; ALMEIDA, 2003).

Nos estudos realizados, nota-se que há uma grande variação entre a idade de aplicação da desrama, o nível de desrama, a qualidade de sítio dos diferentes povoamentos e a idade de avaliação do crescimento após desrama, o que torna a comparação de resultados mais difícil (ALMEIDA, 2003).

Schneider et al. (1999) e Hoppe e Freddo (2003), estudando o efeito da intensidade de desrama em Pinus elliottii Engelm., verificaram que o aumento da intensidade de desrama influencia negativamente a produtividade das florestas e que desramas até $40 \%$ em relação à altura total das árvores proporcionaram os maiores crescimentos.

Resultado parecido foi encontrado por Pires et al. (2002), que avaliaram o crescimento de Eucalyptus grandis submetido a intensidades de desrama artificial de 0\%, 12,5\%, 25\%, 50\% e 75\%, em um povoamento na idade de 11 meses, situado na região de Dionísio (MG), em que as árvores foram originadas de sementes. Os autores concluíram que a desrama artificial afetou negativamente o crescimento das plantas em diâmetro e altura. A desrama artificial de $75 \%$ da copa viva comprometeu significativamente o crescimento em diâmetro, altura e volume das plantas, e ainda foi verificada mortalidade de várias plantas em consequência da redução drástica da superfície fotossintética ativa. $\mathrm{O}$ estudo mostrou que essa intensidade de desrama é inviável em povoamentos comerciais.

Entretanto, Finger et al. (2001) analisaram o efeito da intensidade de desrama no crescimento e produção de um povoamento monoclonal de Eucalyptus saligna com idade de dois anos sob espaçamento de 4 x 1,5 m, no município de Eldorado do Sul (RS). Nesse trabalho, avaliaram-se as intensidades de 0\%, $40 \%, 60 \%$ e $80 \%$ de desrama em relação à altura total das árvores. Foram feitas medições anuais até a idade de 5 anos e observou-se que nesse caso não houve diminuição de diâmetro, altura ou volume de madeira. Observou-se que houve ainda a recuperação da dimensão da copa das árvores com o crescimento em altura já no primeiro ano após a desrama, devido à grande dominância apical e à pouca idade do Eucalyptus saligna nesse experimento.

Outro fator que pode afetar o crescimento da planta é sua posição fitossociológica no povoamento. Segundo Paiva et al. (2001), as árvores de um povoamento podem ser classificadas, de acordo com suas copas, como dominantes, codominantes, intermediárias e suprimidas.

As árvores dominantes geralmente apresentam sua copa acima do nível geral da cobertura, recebendo luz direta de cima e parcialmente dos lados. São geralmente as maiores árvores do povoamento. As codominantes são árvores cujas copas formam o nível geral de cobertura, recebendo luz diretamente de cima e comparativamente pouca luz lateral, apresentando em geral copas de tamanho médio, mais ou menos comprimida sobre os lados. As intermediárias, como o nome indica, são árvores menores que as codominantes, recebem um pouco de luz direta de cima e nenhuma luz lateral. Finalmente, as suprimidas, árvores com copas inteiramente abaixo do nível geral de cobertura, que não recebem luz direta de cima e nem dos lados (PAIVA et al., 2001). 
As duas primeiras classes são árvores mais vigorosas, que apresentam melhor desenvolvimento, ocupam as posições mais altas na cobertura das copas e normalmente têm melhores chances de sobreviver à competição pelos fatores de crescimento, enquanto que a última classe congrega árvores menos vigorosas, que ocupam posições mais baixas de cobertura até que morram (PAIVA et al., 2001).

\section{MATERIAL E MÉTODOS}

\section{Localização e caracterização da área}

O experimento foi instalado no sítio Bom Destino, Distrito de São José das Torres, município de Mimoso do Sul, estado do Espírito Santo, à margem esquerda da BR 101 - Sul, no km 442, sentido Vitória (ES) - Rio de Janeiro (RJ), localizado a $21^{\circ} 06^{\prime} 14^{\prime \prime}$ de latitude sul e $41^{\circ} 13^{\prime} 44,28^{\prime \prime}$ de longitude oeste do Meridiano de Greenwich.

O clima da região, segundo a classificação de Köppen, é do tipo $A w$, tropical chuvoso. A temperatura média anual é superior a $22^{\circ} \mathrm{C}$, com pluviosidade média em torno de $1.000 \mathrm{~mm}$ anuais, com estação seca bem definida (EUCLYDES et al., 2007). O relevo é formado por áreas montanhosas permeadas com áreas planas (baixadas). A área em que se encontra o povoamento está a aproximadamente 80 metros acima do nível do mar.

O povoamento possui uma área de $7,5 \mathrm{ha}$, com inclinação variando entre 30 e $50 \%$. O solo predominante da região é o Latossolo Vermelho-Amarelo distrófico (SIQUEIRA et al., 2004). É composto pelo híbrido Eucalyptus grandis $x$ E. urophylla proveniente de sementes, com espaçamento de $3 \times 2 \mathrm{~m}$, sendo que a primeira desrama artificial ocorreu aos 24 meses de idade.

O plantio foi realizado através do coveamento manual. No momento do plantio foi feita calagem, e no primeiro ano foram realizados os tratos silviculturais de coroamento, roçadas e combate a formigas.

\section{Delineamento experimental}

$\mathrm{O}$ experimento foi instalado em um esquema fatorial $4 \times 4$, sendo 4 classes de altura (A0 - menor que $2 \mathrm{~m}$; A1 - entre 2 e $4 \mathrm{~m}$; A2 - entre 4 e 6 m; e A3 - acima de 6 metros) e 4 intensidades de desrama (I0 - sem realização de desrama; I1 - 40\% de desrama em relação à altura da árvore; I2 - 60\% de desrama em relação à altura da árvore; e I3 - 80\% de desrama em relação à altura da árvore), num delineamento inteiramente casualizado em que foram medidas cinco árvores para cada tratamento e realizadas três repetições, totalizando 15 árvores em cada um dos 16 tratamentos. Um total de 240 árvores foram utilizadas no experimento.

\section{Coleta dos dados}

Para selecionar as árvores de cada parcela, sorteava-se a classe de altura e a intensidade de desrama que seria realizada. Para diminuir o efeito de borda, entrava-se na fila pela estrada do interior do talhão e a partir da terceira árvore verificava-se se a árvore se enquadrava na classe de altura sorteada. Caso se enquadrasse, realizava-se a desrama na intensidade previamente sorteada, tomando-se o cuidado de não desramar árvores vizinhas. Assim, ao selecionar todas as árvores de uma parcela, realizava-se novo sorteio para definir qual tratamento seria realizado na parcela seguinte. Com isso, as parcelas não tinham tamanhos iguais, o que se justifica pelo fato de o plantio ser muito heterogêneo, fazendo com que as classes de altura estejam distribuídas de forma irregular no povoamento.

A circunferência à altura do solo (CAS) foi medida com auxílio de uma fita métrica e posteriormente transformada para diâmetro à altura do solo (DAS), e a altura total de cada árvore foi medida com uma régua telescópica. Outra variável analisada foi a presença de danos físicos e ataques de pragas ou doenças, anotando-se os respectivos códigos, conforme as seguintes escalas: $0=$ sem dano ou ataque; 1 = pequenos danos ou ataques; $2=$ danos ou ataques intermediários; $3=$ sobrevivência por danos ou ataques comprometida. Essa avaliação foi realizada tanto no momento da instalação do experimento quanto na segunda medição. A desrama artificial das árvores foi realizada utilizando-se tesouras de poda, podão e serra de poda.

Seis meses após a intervenção da desrama artificial, foi realizada uma nova observação da fitossanidade das árvores e mensuração das variáveis altura total e DAS, para que essas novas medidas fossem comparadas com as medidas iniciais e assim proporcionassem uma resposta a respeito da intensidade de desrama mais indicada para plantios do híbrido Eucalyptus grandis x E. urophylla. 


\section{Modelo estatístico e análise dos dados}

O modelo estatístico correspondeu a $Y_{\mathrm{ijk}}=\mu+A_{\mathrm{i}}+\mathrm{D}_{\mathrm{j}}+(\mathrm{AD})_{\mathrm{ij}}+\varepsilon_{\mathrm{ijk}}$ em que: $\mathrm{Y}_{\mathrm{ijk}}=$ observação do fator $i$, no nível $j$, na repetição $k ; \mu=$ média geral; $A_{i}=$ efeito da altura $(i=0,1,2$ e 3$) ; D_{j}=$ efeito da desrama $(\mathrm{j}=0,1,2$ e 3$) ; \mathrm{AD}_{\mathrm{ij}}=$ efeito da interação altura e intensidade de desrama; $\epsilon_{\mathrm{ijk}}=$ erro associado a cada observação.

Os efeitos principais e interações (tratamentos) foram submetidos à análise de variância $(\mathrm{F}<0,05)$ e as médias dos tratamentos comparadas pelo teste de Tukey $(\mathrm{P}<0,05)$, descritos por Vieira e Hoffmann (1989), com o uso do software estatístico SAEG (RIBEIRO JR., 2001).

\section{RESULTADOS E DISCUSSÃO}

A análise estatística indicou que os efeitos isolados da classe de altura da planta e da intensidade de desrama sobre o crescimento foram significativos, enquanto a interação entre esses fatores não apresentou tal efeito sobre o crescimento ao nível de 5\% de probabilidade (Tabela 1).

Tabela 1. Resumo da análise de variância para o diâmetro à altura do solo (DAS) e altura total das árvores (Ht) de acordo com a classe de altura e a intensidade de desrama.

Table 1. Summary of variance analysis for diameter at soil height (DSH) and total tree height (TH) according to height class and intensity of pruning.

\begin{tabular}{lcc}
\hline Fonte de variação & DAS $(\mathbf{c m})$ & Ht $(\mathbf{m})$ \\
\hline Classe de altura & $19,62^{*}$ & $50,34^{*}$ \\
Desrama & $4,91 *$ & $4,95^{*}$ \\
Classe de altura x desrama & 1,62 & 1,100 \\
\hline *: significativo ao nível de 5\% de probabilidade; DAS: diâmetro a altura do solo; Ht: altura total.
\end{tabular}

Na tabela 2 estão apresentados os valores médios de crescimento do diâmetro à altura do solo e altura total obtidos para cada classe de altura da planta e intensidade de desrama adotada. A tabela apresenta ainda a diferença entre as classes de fitossanidade das plantas entre as duas medições. Nota-se ainda que a condição fitossanitária de nenhuma das árvores foi alterada no experimento, o que indica que essa variável não se encontra relacionada com a intensidade de desrama artificial ou com a classe de altura da árvore.

Tabea 2. Média das variáveis dendrométricas observados em cada tratamento.

Table 2. Average of dendrometric variables in each treatment.

\begin{tabular}{lcccc}
\hline Classe de altura & Int. desrama & DAS $(\mathbf{c m})$ & Ht $(\mathbf{m})$ & FIT \\
\hline A3 & I0 & 3,36135 & 4,0 & 0 \\
A3 & I1 & 2,52261 & 3,1 & 0 \\
A2 & I0 & 2,06901 & 3,4 & 0 \\
A3 & I2 & 2,00535 & 4,1 & 0 \\
A2 & I1 & 1,97352 & 3,3 & 0 \\
A3 & I3 & 1,46423 & 2,6 & 0 \\
A1 & I2 & 1,41330 & 1,9 & 0 \\
A2 & I2 & 1,22231 & 3,2 & 0 \\
A1 & I1 & 1,02496 & 2,0 & 0 \\
A1 & I0 & 0,97403 & 2,4 & 0 \\
A0 & I1 & 0,80002 & 1,1 & 0 \\
A2 & I3 & 0,73211 & 2,6 & 0 \\
A0 & I2 & 0,70736 & 1,1 & 0 \\
A0 & I0 & 0,56323 & 0,8 & 0 \\
A1 & I3 & 0,44563 & 1,7 & 0 \\
A0 & I3 & 0,38834 & 0,7 & 0 \\
\hline C1
\end{tabular}

Classes de altura: A0: menores que 2 metros; A1: 2 a 4 metros; A2: 4 a 6 metros; A3: acima de 6 metros. Intensidade de desrama: I0: sem realização de desrama; I1: 40\% de desrama em relação à altura da árvore; I2: 60\% de desrama em relação à altura da árvore; I3: $80 \%$ de desrama em relação à altura da árvore; DAS: diâmetro à altura do solo; Ht: altura total; FIT: diferença entre classe de fitossanidade das plantas entre a primeira e a segunda medição. 
Nota-se uma tendência de árvores maiores crescerem mais em relação às árvores situadas em estratos inferiores do povoamento (Tabela 3 ). Tal efeito é explicado principalmente devido às classes de altura estarem relacionadas diretamente à posição fitossociológica da planta no povoamento, confirmando a hipótese de que as árvores dominantes crescem mais que árvores suprimidas, devido a diversos fatores, como competição por luz, água, nutrientes ou pelo material genético das árvores maiores serem mais adaptados ao ambiente que o das árvores menores.

Tabela 3. Teste de médias para o efeito da classe de altura sobre o crescimento das árvores para as variáveis dendrométricas DAS e Ht.

Table 3. Average test for effect of height class on tree growth for dendrometric variables DSH and TH.

\begin{tabular}{lcc}
\hline Classe de altura & DAS $(\mathbf{c m})$ & Ht $(\mathbf{m})$ \\
\hline $\mathrm{A} 0$ & $0,6163 \mathrm{C}$ & $1,01 \mathrm{c}$ \\
$\mathrm{A} 1$ & $0,9386 \mathrm{BC}$ & $1,96 \mathrm{~b}$ \\
$\mathrm{~A} 2$ & $1,6473 \mathrm{AB}$ & $3,15 \mathrm{a}$ \\
$\mathrm{A} 3$ & $2,4067 \mathrm{~A}$ & $3,5 \mathrm{a}$ \\
\hline Classes de altura: A0: menores que 2 metros; A1: 2 a 4 metros; A2: 4 a 6 metros; A3: acima de 6
\end{tabular}

Referindo-se ao efeito da intensidade de desrama, observou-se haver uma tendência de árvores menos desramadas apresentarem maior crescimento, tanto em diâmetro quanto em altura (Tabela 4).

Tal tendência é também observada nos trabalhos realizados por Hoppe e Freddo (2003), Pires et al. (2002) e Schneider et al.(1999), que mostraram redução no crescimento em altura e diâmetro de acordo com o aumento da intensidade de desrama sobre a planta. Por outro lado, os resultados divergiram dos encontrados por Finger et al. (2001), em que não foi encontrado efeito da intensidade de desrama sobre o crescimento das árvores.

Tabela 4. Teste de médias para o efeito da intensidade de desrama sobre o crescimento das árvores para as variáveis dendrométricas DAS e Ht.

Table 4. Average test for pruning intensity on tree growth for dendrometric variables DAS and Ht.

\begin{tabular}{lcc}
\hline Intensidade de desrama & DAS $(\mathbf{c m})$ & $\mathbf{H t}(\mathbf{m})$ \\
\hline I0 & $1,7946 \mathrm{~A}$ & $2,68 \mathrm{a}$ \\
I1 & $1,5667 \mathrm{~A}$ & $2,60 \mathrm{a}$ \\
I2 & $1,3881 \mathrm{AB}$ & $2,45 \mathrm{ab}$ \\
I3 & $0,8594 \mathrm{~B}$ & $1,89 \mathrm{~b}$ \\
\hline
\end{tabular}

Intensidade de desrama: I0: sem realização de desrama; I1: 40\% de desrama em relação à altura da árvore; I2: 60\% de desrama em relação à altura da árvore; I3: 80\% de desrama em relação à altura da árvore; DAS: diâmetro à altura do solo; Ht: altura total.

Nos trabalhos consultados, as intensidades de desrama até $40 \%$ em relação à altura total da árvore não divergiram estatisticamente entre si e apresentaram crescimentos superiores aos tratamentos com intensidade de desrama acima de $40 \%$. Entretanto, no presente trabalho, a intensidade de $60 \%$ de desrama não demonstrou diferença entre a testemunha e o tratamento de $40 \%$ de desrama artificial, embora tenha sido percebida uma tendência de esse tratamento proporcionar crescimento menor às árvores. A diferença de crescimento entre as classes de desrama deverá ser mais visível com o aumento da idade do povoamento e poderá ser observada em medições futuras.

Como em nenhum dos tratamentos notou-se mortalidade ou alteração nas condições fitossanitárias das árvores, esses fatores não parecem estar ligados à intensidade de desrama ou à classe de altura. Entretanto, alguns autores defendem que árvores menores tendem a apresentar maior mortalidade, fato que, possivelmente, poderá ser analisado com o passar do tempo.

\section{CONCLUSÕES}

- Os fatores intensidade de desrama e classe de altura não exercem efeito sobre a mortalidade ou fitossanidade das árvores.

- Há uma tendência de redução do crescimento com o aumento da intensidade de desrama. As intensidades de desrama de $0,40 \%$ e $60 \%$ não apresentam diferença estatisticamente significativa no 
crescimento das árvores do povoamento, embora haja uma tendência da intensidade de 60\% proporcionar um crescimento menor que as demais, o que deve ser comprovado em medições posteriores. Já a desrama realizada a uma intensidade de $80 \%$ em relação à altura da árvore apresentou crescimento inferior à testemunha e ao tratamento de intensidade $40 \%$.

- Árvores situadas nas maiores classes de altura apresentam crescimento mais intenso que árvores localizadas em extratos inferiores.

- A interação da intensidade de desrama com a classe de altura das árvores não apresenta efeito significativo sobre o crescimento ao nível de $5 \%$ de probabilidade de confiança.

\section{REFERÊNCIAS}

ALMEIDA, M. L. Desrama artificial em clones de Eucalyptus grandis $\mathbf{x}$ E. urophylla com diferentes arquiteturas de copa. 2003. 119 f. Dissertação (Mestrado em Ciências Florestais) - Universidade Federal de Viçosa. 2003.

ASSOCIAÇÃO BRASILEIRA DE PRODUTORES DE FLORESTAS PLANTADAS (ABRAF). Anuário Estatístico da ABRAF 2010: Ano base 2009. Brasília, DF, 140 p. 2010.

EUCLYDES, H. P.; FERREIRA, P. A.; FARIA FILHO, R. F.; SANTOS, A. P.; Regionalização hidrológica na região hidrográfica capixaba, compreendida entre os limites da bacia do rio Doce e do rio Itabapoana. In: SIMPÓSIO DE RECURSOS HÍDRICOS DO NORTE E CENTRO-OESTE, 1., 2007, Cuiabá. Anais... Associação Brasileira de Recursos Hídricos, 2007.

FINGER, C. A. G.; SCHEIDER, P. R.; BAZZO, J. L.; KLEIN, J. E. M. K. Efeito da intensidade de desrama sobre o crescimento e a produção de Eucalyptus saligna Smith. Cerne, Lavras, v. 7, n. 2, p. 53 64, 2001.

HOPPE, J. M.; FREDDO, A. R. Efeito da intensidade de desrama na produção de Pinus elliottii Engelm. no município de Piratini, RS. Ciência Florestal, Santa Maria, v. 13, n. 2, p. 47 - 56, 2003.

HOSOKAWA, R. T.; MOURA, J. B.; CUNHA, U. S. Introdução ao manejo e economia de florestas. Curitiba: UFPR, 1998. 162 p.

KRAMER, P. J.; KOZLOWSKI, T. T. Fisiologia das árvores. Lisboa: Fundação Calouste-Gulbenkian, 1972. $638 \mathrm{p}$.

MONTE, M. A.; REIS, M. G. F.; REIS, G. G.; LEITE, H. G.; CACAU, F. V.; ALVES, F. F. Crescimento de um clone de eucalipto submetido a desrama e desbaste. Revista Árvore, Viçosa, v. 33, n. 5, p. 777 787, 2009.

PAIVA, H. N.; JACOVINE, L. A. G.; RIBEIRO, G. T.; TRINDADE, C. Cultivo de eucalipto em propriedades rurais. Viçosa: Aprenda Fácil. 2001. 138 p.

PIRES, B. M.; REIS, M. G. F.; REIS, G. G. Crescimento de Eucalyptus grandis submetido a diferentes intensidades de desrama artificial na região de Dionísio, MG. Brasil Florestal, Brasília, n. 73, p. 13 - 21, 2002.

POLLI, H. Q. Crescimento e qualidade da madeira para serraria em clone de Eucalyptus grandis [Hill ex Maiden] submetido a desrama artificial. 97 f. Dissertação (Mestrado em Ciência Florestal) Universidade Federal de Viçosa, Viçosa, 2005.

PULROLNIK, K. Crescimento, dinâmica de copa e qualidade da madeira para serraria de clone de Eucalyptus grandis [Hill ex Maiden] submetido a desrama artificial. 2002. 96 f. Dissertação (Mestrado em Ciências Florestais) - Universidade Federal de Viçosa, Viçosa, 2002.

REZENDE, J. L. P.; PÁDUA, C. T. J.; OLIVEIRA, A. D.; SCOLFORO, J. R. S. Análise econômica de fomento florestal com eucalipto no estado de Minas Gerais. Cerne, Lavras, v. 12, n. 3, p. 221 - 231, 2006.

RIBEIRO JÚNIOR, I. Análises estatísticas no SAEG. Viçosa: UFV, 2001. 301 p. 
SCHETTINO, L. F.; SOUZA, A. L.; SILVA, M. L.; BRAGA, G. M.; REZENDE, J. L. P.; SOUZA, A. P. Diagnóstico para a gestão florestal sustentável no Espírito Santo. Revista Árvore, Viçosa, v. 24, n. 4, p. 445 - 456, 2000.

SCHNEIDER, P. R.; FINGER, C. A. G.; HOPPE, J. M. Efeito da intensidade de desrama na produção de Pinus elliottii Engelm. implantado em solo pobre, no estado do Rio Grande do Sul. Ciência Florestal, Santa Maria, v. 9, n. 1, p. 35 - 46, 1999.

VALE, R. S. Efeito da desrama artificial no crescimento e na qualidade da madeira de clones de eucalipto, em sistema agro-silvo-pastoril. 2000. 96 f. Dissertação (Mestrado em Ciência Florestal) Universidade Federal de Lavras, Lavras, 2000.

VALE, R. S.; MACEDO, R. L. G.; VENTURIN, N.; MORI, F. A.; MORAIS, A. R. Efeito da desrama artificial na qualidade da madeira de clones de eucalipto em sistema agrossilvipastoril. Revista Árvore, Viçosa, MG, v. 26, n. 3, p. 285 - 297, 2002.

VIEIRA, S.; HOFFMANN, R. Estatística experimental. São Paulo: Atlas, 1989. 179 p. 